\title{
The differences in functional recovery between HIV-positive and HIV-negative stroke survivors
}

\author{
Jenny Janse van Rensburg @i), Witness Mudzi@, Veronica Ntsiea@ \\ Department of Physiotherapy, School of Therapeutic Sciences, Faculty of Health Sciences, University of the Witwatersrand, Johannesburg, South Africa
}

Received: May 15, 2017 Accepted: February 05, 2018 Published online: November 03, 2018

\begin{abstract}
Objectives: This study aims to identify the differences in functional abilities between stroke survivors who are human immunodeficiency virus (HIV)-positive and HIV-negative.

Patients and methods: This was a retrospective, longitudinal record review of stroke survivors' files between April 2005 and December 2010. Of a total of 173 stroke survivors who were admitted to the rehabilitation unit, 141 (75 males, 66 females; mean age $52.7 \pm 14.3$ years; range, 19 to 86 years) met the inclusion criteria. The patients were divided into two groups as HIV-positive ( $\mathrm{n}=21)$ and HIV-negative ( $\mathrm{n}=120$ ). Functional ability was recorded using the admission and discharge BETA ${ }^{\circledast}$ scores.

Results: Ischemic strokes were more prevalent than hemorrhagic strokes ( $74.5 \%$ vs. $25.5 \%$, respectively) with hypertension as the most common (31.9\%) stroke risk factor. The mean age of stroke onset for HIV-positive patients and HIV-negative patients was 39.6 years and 54.9 years, respectively. In HIV-positive patients, the mean duration of rehabilitation was 7.5-day shorter than HIV-negative patients. After receiving rehabilitation from a multidisciplinary team, the HIV-positive group improved with a mean of 40 points and the HIV-negative group improved with a mean of 38 points. The similarities in functional outcome between the HIV-positive and HIV-negative group were related to the fact that HIV-positive stroke survivors were relatively younger than the HIV-negative group.

Conclusion: Our study results show that patients who sustain a stroke, are HIV-positive, are receiving antiretroviral therapy and rehabilitation may recover similar to those who are HIV-negative, spending a similar length of stay in a rehabilitation clinic. Therefore, stroke survivors who are HIV-positive should receive full rehabilitation similar to any other stroke survivors.
\end{abstract}

Keywords: Comorbidity; functional ability; human immunodeficiency virus; stroke.

Stroke is a significant contributor to disease worldwide $^{[1]}$ and is the second leading cause of death in both men and women. ${ }^{[2]}$ It is not only a common cause of mortality, but also of morbidity. In general, stroke incidence is higher in the Sub-Saharan Africa than in developed countries. ${ }^{[3]}$ Hypertension, diabetes, obesity, and hypercholesterolemia are known risk factors of stroke to which prevention has been focused; however, viral infections appear to be an emerging risk factor of stroke. ${ }^{[4]}$

Post-stroke recovery involves complex rehabilitation and, thus, differs between individuals. The coexistence of psychosocial and cognitive impairments, as well as a broad range of other neurological and sociodemographic factors has been seen to affect the functional recovery of stroke survivors. ${ }^{[5]}$ The main goal of stroke rehabilitation is to help the patient to achieve his/her highest physical and psychological performance, with the ultimate goal of a stroke survivor being one of functional independence allowing them to return to home and reintegrate back into community. ${ }^{[6]}$

There are 35 million individuals with human immunodeficiency virus (HIV) of which 24.7 million are living in the Sub-Saharan Africa. ${ }^{[7]}$ Although new cases of HIV infections tend to decline, 2.1 million new infection cases have been reported in 2013, indicating a $38 \%$ decline as of $2001 .^{[7]}$ Almost half of all individuals with HIV are aware of their disease. ${ }^{[7]}$ Approximately $86 \%$ of HIV-infected patients who are aware of their disease in the Sub-Saharan Africa are on antiretroviral

Corresponding author: Veronica Ntsiea, PhD. Physiotherapy department Faculty of Health Sciences University of the Witwatersrand 7 York Road, Parktown, 2193 Johannesburg, South Africa. e-mail: Veronica.Ntsiea@wits.ac.za 
therapy (ART) ${ }^{[7]}$ South Africa is regarded as having the world's largest population of individuals with acquired immunodeficiency syndrome (AIDS), with a prevalence of $18 \%$ recorded by the Joint United Nations Programme on HIV/AIDS (UNAIDS) Global Report $^{[8]}$ and 6.7 to 7.4 million as reported by the UNAIDS ${ }^{[9]}$ HIV estimates according to each country. An estimated 2.1 million individuals became infected with HIV in 2013 worldwide. ${ }^{[7]}$ An individual with HIV would go through periods of both wellness and illness, but HIV always has a physical, psychological, and social impact. ${ }^{[10]}$

There is a higher prevalence of cerebrovascular risk factors in HIV-infected individuals. ${ }^{[1]}$ This increase is believed to be caused by the negative effects of ART, increasing age and the direct impact of the HIV itself. ${ }^{[1]}$ Early-onset atherosclerosis, diabetes, lipodystrophy, hypertension, and myocardial infarction have been linked to ART treatment. ${ }^{[12]}$ Neurological symptoms are the initial manifestations of HIV infection in about 10 to $20 \%$ of patients. ${ }^{[13]}$ Among the HIV-infected population, the male-tofemale ratio of stroke is 1.5:1 with a mean age of 32.1 years in the female population. Ninety-four percent of these strokes are cerebral infarcts and $6 \%$ are intracranial cerebral hemorrhages with a younger population being affected by HIV (Mochan et al. 2003). ${ }^{[14]}$ A variety of factors may affect functional outcome in strokes survivors affected by HIV.

In a study done by Hama et al., ${ }^{[15]}$ depression and apathy were found to be predictors of poor functional post-stroke recovery. Motivational levels to participate in the rehabilitation process may also be affected due to depression. ${ }^{[15]}$ Side effects of medication may also affect functional outcome. In addition, HIVinfected individuals are treated using the highly active antiretroviral therapy (HAART) in whom side-effects may indirectly affect their quality of life. ${ }^{[15]}$ With the introduction of ART drugs, HIV-related mortality has been reduced, although this life-extending treatment has also certain side-effects which may affect the quality of life. ${ }^{[15]}$

Rusch et al. ${ }^{[1]}$ identified the 'cycles of illness and wellness' for those on treatment, but more importantly, they found that infected individuals' quality of life was affected in physical, psychological, and social spheres of life. A large number of individuals infected with HIV are also found to suffer from anemia resulting in weakness and fatigue. ${ }^{[15]}$ All comorbidities may result in reduced energy levels, weakness, and poor motivation, thereby, affecting the individual's recovery. Furthermore, the virus may have an impact on the digestive tract ${ }^{[16]}$ leading to a high prevalence of gastrointestinal disorders in HIV-infected patients. Some individuals also seem to have difficulty in performing activities of daily living due to poor nutrition. ${ }^{[16]}$

Post-stroke recovery largely depends on stroke type and the existence of a comorbid disease and, as previously discussed, the effect of HIV on the body may hamper recovery severely. ${ }^{[17]}$ Pain is also an important and challenging symptom of HIV, often resulting in painful sensory neuropathy and myopathy which, if not treated correctly, may lead to chronic pain and hamper participation in rehabilitation. ${ }^{[13]}$ Therefore, a stroke survivor with HIV may have more comorbidities which may affect their functional outcome.

In the present study, we aimed to evaluate the differences in functional status between HIV-positive and HIV-negative stroke survivors.

\section{PATIENTS AND METHODS}

This was a retrospective, longitudinal record review of stroke survivors' medical files. Participants for the study were drawn from a rehabilitation unit in the North West province of South Africa. The North West Province is completely landlocked, making up $0.5 \%$ of South Africa and is reported as being one of the poorer provinces in South Africa. ${ }^{[18]}$ In the 2001 census, $20 \%$ of the population who was $\geq 20$ years old had no formal schooling. ${ }^{[18]}$ About $69 \%$ of all households in the province live in formal dwellings; $22 \%$ in informal structures, and $5 \%$ in traditional structures. ${ }^{[18]}$ The rehabilitation unit used for this study serves the whole of the North West province, as they are the only physical rehabilitation unit in the province.

The study was conducted between April 2005 and December 2010. This time period was chosen, as the rehabilitation unit was using the South African Database for Functional Medicine $\left(\right.$ BETA $^{\circ}[\beta]$ assessment tool) to keep record of patients' progress at the time. Throughout the study period, there were 570 admissions to the unit of which 173 were stroke survivors. However, 32 participants' files did not meet the inclusion criteria, thus leaving a study group of 141 participants' files. Information was captured from these files and, then, analyzed. Participants for the study were selected, if they had the first incident stroke and functional ability was well-documented using the BETA $^{\star}$ tool from admission to discharge. Patients with an unknown HIV status were excluded. 
Of a total of 173 stroke survivors who were admitted to the rehabilitation unit, 141 (75 males, 66 females; mean age $52.7 \pm 14.3$ years; range, 19 to 86 years) met the inclusion criteria. The patients were divided into two groups as HIV-positive $(\mathrm{n}=21)$ and HIV-negative $(n=120)$.

The study protocol was approved by the Human Research Ethics Committee of the University of the Witwatersrand (No. M110493). A written informed consent was obtained from each participant. Participant confidentiality was ensured at all times during data collection by not writing participants' names on the data collection forms. File numbers were used on the coding sheets to maintain confidentiality. The study was conducted in accordance with the principles of the Declaration of Helsinki.

\section{Instrumentation and outcome measures}

The BETA ${ }^{\oplus}$ tool was used to determine participants' functional ability. The $\beta$ assessment tool is one of three platform level tools designed by the South African Database for Functional Medicine (SADFM). It allows the assessor to monitor a participants' progress, as it is reported on a weekly basis. ${ }^{[19]}$ It is based on the well-known American version, the Functional Independence Measure. This tool works within the World Health Organization framework of the International Classification of Function, Disability, and Health. ${ }^{[19]}$ Patients are scored by a multi-professional team, covering 18 areas with points given between one and seven. Thirteen of the items are physical domains and five are cognition items. Functional ability is assessed on the basis of direct observation. Evaluation domains include eating; grooming; bathing; dressing upper body; dressing lower body; toileting; bladder management; bowel management; transfers: bed, chair, wheelchair; transfers: toilet; transfers: tub, shower; locomotion: walking, wheelchair; locomotion: stairs; comprehension; expression; social interaction; problem solving and memory. ${ }^{[19]}$ The higher the $\beta$-score, the greater the functional ability. A normal score for a fully functional individual is $126 / 126 .{ }^{[19]}$

The participants' demographic and clinical characteristics were captured using a self-designed questionnaire. Data including age, gender, weight, race, hospital length of stay, number of strokes, stroke subtype, existing comorbidities, date of admission, date of discharge, date of stroke, HIV status, and whether they were receiving ART drugs and their latest CD4 count were recorded.

\section{Statistical analysis}

The data collected from the total sample size $(\mathrm{n}=141)$ was first analyzed using descriptive statistical tests to describe the group demographics and clinical characteristics. The Shapiro-Wilk expanded test was performed to analyze whether the distribution

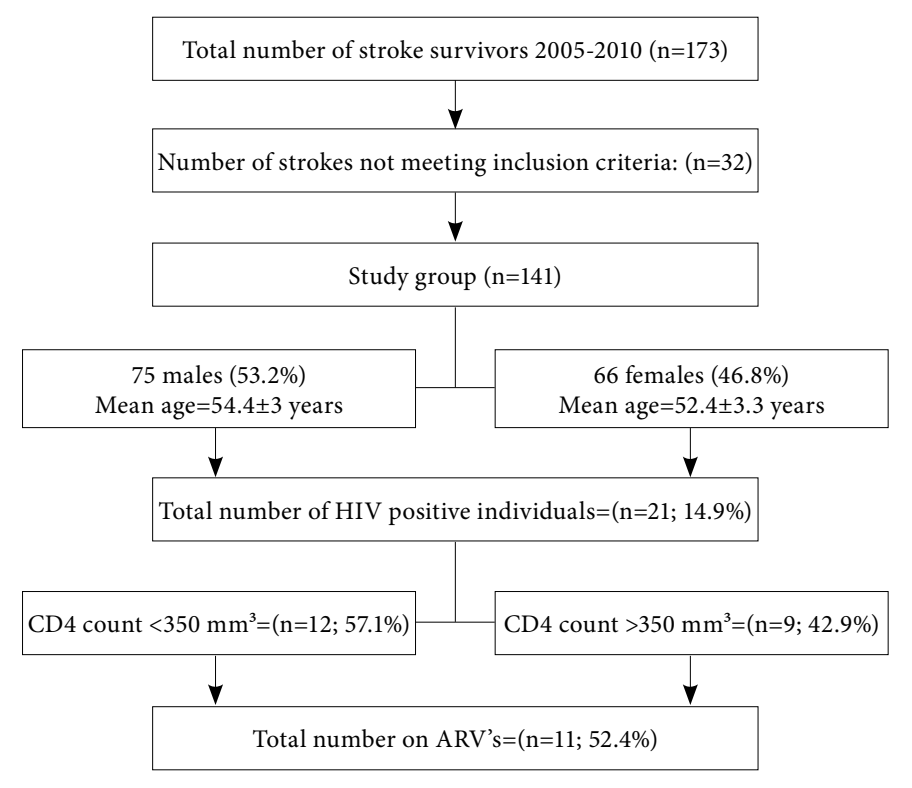

Figure 1. Sex, HIV status, and CD4 count of study population. HIV: Human immunodeficiency virus; CD4: Cluster of differentiation 4; ARV: Antiretroviral. 
Table 1. Demographic and clinical characteristics: Categorical variables

\begin{tabular}{|c|c|c|c|c|c|c|}
\hline & \multicolumn{2}{|c|}{ HIV positive $(n=21)$} & \multicolumn{2}{|c|}{ HIV negative $(n=120)$} & \multicolumn{2}{|c|}{ Total $(\mathrm{n}=141)$} \\
\hline & $\mathrm{n}$ & $\%$ & $\mathrm{n}$ & $\%$ & $\mathrm{n}$ & $\%$ \\
\hline \multicolumn{7}{|l|}{ Gender } \\
\hline Male & 8 & 38.1 & 67 & 55.8 & 75 & 53.2 \\
\hline Female & 13 & 61.9 & 53 & 44.2 & 66 & 46.8 \\
\hline \multicolumn{7}{|l|}{ Race } \\
\hline African & 21 & 100.0 & 80 & 66.7 & 101 & 71.6 \\
\hline Caucasian & 0 & 0.0 & 33 & 27.5 & 33 & 23.4 \\
\hline Colored & 0 & 0.0 & 6 & 5.0 & 6 & 4.3 \\
\hline Indian & 0 & 0.0 & 1 & 0.9 & 1 & 0.7 \\
\hline \multicolumn{7}{|l|}{ Subtype } \\
\hline Ischemic & 20 & 95.2 & 85 & 70.8 & 105 & 74.5 \\
\hline Hemorrhagic & 1 & 4.8 & 35 & 29.2 & 36 & 5.5 \\
\hline Hypertension & 8 & 34.8 & 37 & 31.4 & 45 & 31.9 \\
\hline Diabetes & 2 & 9.5 & 7 & 5.8 & 9 & 6.4 \\
\hline $\begin{array}{l}\text { Treatment received: } \\
\text { Medical officer, } \\
\text { physiotherapist, occupational } \\
\text { therapist, clinical psychologist, } \\
\text { dietician and nursing }\end{array}$ & 21 & 100.0 & 120 & 100.0 & 141 & 100.0 \\
\hline
\end{tabular}

HIV: Human immunodeficiency virus.

of the sample means was normal. Categorical data were expressed in number and percentage, while continuous data were expressed in mean \pm standard deviation (SD). The chi-square and Fisher's exact tests were used to compare categorical demographic and clinical characteristics between the HIV-positive and HIV-negative patients. A two-sample t-test was used to compare continuous demographic and clinical characteristics between the HIV-negative and HIV-positive patients, mean admission and discharge functional scores between the HIV-negative and HIV-positive patients, and to compare the functional change in ability of those who were HIV-positive and those who were HIV-negative at discharge. Statistical analysis was performed using the STATA 10 software version 10.2 software (STATA Corp., College Station, TX, USA). A $p$ value of $<0.05$ was considered statistically significant with a confidence interval of $95 \%$.

\section{RESULTS}

There was no statistically significant difference in the demographic and clinical characteristics between the HIV-positive and HIV-negative patients. Figure 1 shows a description of the study group's gender, HIV status, and CD4 count. About 57\% of the stroke survivors who were HIV-positive had a CD4 count below 350 cells $/ \mathrm{mm}^{3}$.

The demographic details and clinical characteristics of the categorical variables of the study group are shown in Table 1. Ischemic strokes were more prevalent than hemorrhagic strokes $(74.5 \%$ vs. $25.5 \%$ respectively), and hypertension (31.9\%) was the most common stroke risk factor. The demographic details and clinical characteristics of the continuous variables of the study group are shown in Table 2. There was a discrepancy in age of stroke onset between the

Table 2. Demographic and clinical characteristics: Continuous variables

\begin{tabular}{|c|c|c|c|}
\hline & HIV positive $(\mathrm{n}=21)$ & HIV negative $(\mathrm{n}=120)$ & Total $(\mathrm{n}=141)$ \\
\hline & Mean \pm SD & Mean \pm SD & Mean \pm SD \\
\hline Age (year) & $39.6 \pm 10.8$ & $54.9 \pm 13.6$ & $52.7 \pm 14.3$ \\
\hline Length of hospital stay (days) & $30.5 \pm 8.8$ & $38 \pm 15.6$ & $36.9 \pm 15.1$ \\
\hline Weight (kg) & $58.4 \pm 16.3$ & $67.1 \pm 16.8$ & $65.8 \pm 17.0$ \\
\hline $\begin{array}{l}\text { Stroke duration prior to admission to } \\
\text { Witrand hospital (days) }\end{array}$ & $26.2 \pm 14.4$ & $25.7 \pm 24.0$ & $25.8 \pm 22.7$ \\
\hline
\end{tabular}

HIV: Human immunodeficiency virus; SD: Standard deviation. 
Table 3. Functional ability based on mean BETA ${ }^{\oplus}$ scores

\begin{tabular}{|c|c|c|c|}
\hline & HIV positive $(\mathrm{n}=21)$ & HIV negative $(\mathrm{n}=120)$ & $p$ \\
\hline & Mean \pm SD & Mean \pm SD & Mean \pm SD \\
\hline Beta scores on admission & $56 \pm 21$ & $60 \pm 25$ & 0.53 \\
\hline Beta scores at discharge & $96 \pm 28$ & $97 \pm 23$ & 0.60 \\
\hline $\begin{array}{l}\text { Mean Beta scores change from } \\
\text { admission to discharge }\end{array}$ & $40 \pm 19$ & $38 \pm 20$ & 0.88 \\
\hline
\end{tabular}

HIV: Human immunodeficiency virus; SD: Standard deviation; Test: Two sample t test; Significance: $\mathrm{p}<0.05$.

HIV-positive and HIV-negative patients. The mean age of stroke onset in the HIV-positive group was \pm 15 years earlier than those individuals who were HIV-negative. The HIV-negative group required approximately a week longer of rehabilitation intervention. Both groups began their rehabilitation at approximately the same time period after the stroke onset.

The participants' functional abilities were measured using the BETA ${ }^{\circ}$ scores. The results of the functional abilities of the study group on admission, at discharge, and the change in functional ability from admission to discharge are shown in Table 3. The mean BETA scores of the two groups on admission and discharge were not statistically significantly different. The mean change differences in the scores between the two groups were also not statistically significant.

\section{DISCUSSION}

In the present study, the male-to-female ratio was 1.1:1 and $53 \%$ of the participants were male. This finding is almost similar to that by Mochan et al.'s ${ }^{[14]}$ study in which stroke was found in participants with a male-to-female ratio of 1.5:1. However, the results of this study showed that, within the HIV-positive group, more females appeared to be affected by stroke than males (13 females to 8 males) with a male-tofemale ratio of 1:1.6. The high number of HIV-infected females in this study is a reflection of the UNAIDS ${ }^{[9]}$ South African HIV report, indicating that four million of the estimated seven million HIV-infected South Africans were females. However, stroke affects males at an earlier age than females in the general population, with females having stroke approximately 4.3 years later than men. ${ }^{[20]}$ In several areas in the Sub-Saharan Africa, there is a higher prevalence of HIV in young women, possibly as a result of greater to infected partners, or due to their higher risk of acquiring HIV from an infected partner. ${ }^{[21]}$

Of those individuals infected with HIV, 12 individuals (57\%) had a CD4 count of $<350 \mathrm{~mm}^{3}$. In
South Africa, during the study period, ART drugs were administered, when the CD4 count was $\leq 350 \mathrm{~mm}^{3}$. ${ }^{[22]}$ Thus, it is important to note that 11 individuals of the study group were receiving ART drugs. It can, therefore, be extrapolated that possibly the public health sector was implementing this treatment protocol effectively. The introduction of ART drugs into the treatment regimen has the potential to improve the CD4 count which may further prevent the development or onset of other diseases, lessening the health burden to a degree. ${ }^{[23]}$

On the other hand, there is a considerable difference in age of stroke onset, when comparing the individuals who were HIV-positive with those who were HIV-negative. The mean age of stroke onset for an individual with HIV was $39.6 \pm 10.8$ years, whereas the mean age of stroke onset for individuals who did not have HIV was $54.9 \pm 13.6$ years. In a study by Lemogoum et al., ${ }^{[24]}$ it was reported that stroke often affected a younger population in developing countries, compared to developed countries. Similarly, Tobias et al. ${ }^{[25]}$ found that the mean male onset of stroke was 68.9 years and 73.3 years for females, with males having $\pm 20 \%$ higher prevalence rate than females, supporting the older onset of stroke in the Western countries. It should be noted that the mean age of stroke onset was 54.9 years in the HIV-negative group, approximately 14 and 18 years earlier than the mean age of stroke onset in males and females in the Western countries respectively. Thus, although it has been shown that age of stroke onset in developing countries is lower than that of developed countries, it is worth noting that the age of stroke onset in HIV-positive patients is lower than that of HIV-negative individuals within the same country.

The two subtypes of stroke appear in both clinical groups, i.e. the HIV-positive and HIV-negative individuals. Consistent with the literature, ischemic strokes were more common than hemorrhagic strokes with a ratio of 3:1. ${ }^{[20,25-29]}$ The risk for an ischemic stroke is a further three to four-fold higher in individuals 
with hypertension which is important, when one considers the importance of hypertension as a stroke risk factor. ${ }^{[26]}$ Lemogoum et al. ${ }^{[24]}$ found that, despite cerebral hemorrhage being a leading cause of stroke fatality in the Sub-Saharan Africa, cerebral infarction occurred more often. Regarding risk factors for stroke, hypertension far exceeded the others, affecting $34.8 \%$ in the HIV-positive group and $31.4 \%$ in the HIV-negative group. Benjamin et al. ${ }^{[30]}$ found that hypertension affected $80 \%$ of the stroke survivors in their study. Despite the vast difference in numbers within each of the study groups, this study confirmed this trend. Hypertension is the most consistent and powerful predictor of stroke and is involved in approximately $70 \%$ of all strokes. ${ }^{[24]}$ Treatment of hypertension has the potential to reduce stroke risk by 35 to $40 \%$, yet surveys still indicate poor control of hypertension in Africa. ${ }^{[23]}$ Therefore, public education and awareness on stroke risk factors would greatly assist in lessening the social and economic burden of stroke.

Diabetes affected $9.5 \%$ of individuals with HIV, as opposed to $5.8 \%$ of individuals without HIV. This finding validates Young et al.'s ${ }^{[31]}$ findings which indicated that individuals with HIV were at a higher risk of developing diabetes. The rapid leap toward urbanization in the Sub-Saharan Africa has been argued to lead to a rise in the prevalence of diabetes and other cardiovascular diseases. ${ }^{[32]}$

In the present study, we found that the mean length of hospital stay was $30.5 \pm 8.8$ days and $38 \pm 15.6$ days in the HIV-positive and HIV-negative patients, respectively. This discrepancy can be a result of the notably younger HIV-positive group in this study. It can be argued that the advantage of age on recovery in this group may result in gaining, on average, a similar number of points on the BETA ${ }^{\circ}$ scale with those individuals without HIV. It must be mentioned that this gain in points also occurred over a shorter time period. Thus, one could reason that age was the determining factor for more effective and positive outcomes. However, when looking at the bigger picture, a younger population of individuals is being affected by stroke due to their HIV status. This could result in the growth of a younger disabled population, possibly resulting in an increase in the applications for disability grants. If younger individuals are not working, one can assume a negative effect on their work force and labor, if return to work is not possible.

Functional ability was determined by the individual's Beta scores out of 126. Both HIV-positive and HIV-negative individuals were admitted at approximately the same amount of time post-stroke,
26.2 and 25.7 days, respectively. This might have contributed to some extent to slight differences in the functional ability between the two groups.

The mean BETA ${ }^{\oplus}$ scores for individuals with HIV was 56 points and for individuals without HIV was 60 at the time of admission. The recovery of motor function has been documented to occur most effectively during the first month post-stroke, allowing for the improvement of motor deficits. ${ }^{[33,34]}$ In both groups, the rehabilitation process began at approximately the same period of time after stroke. Functional recovery is influenced by an array of biological and environmental factors. ${ }^{[34]}$ Having rehabilitation at a similar timeframe after stroke eliminates any bias toward a specific group.

Age at stroke onset appeared to be the greatest variance between the HIV-negative group and the HIV-positive group. This is more than likely as a result of the age of the group most at risk of developing HIV. ${ }^{[29]}$ Prior to their commencement of rehabilitation, it should be taken into account that neurological recovery requires a degree of brain reorganization and that with age comes a certain degree of neuronal loss. ${ }^{[35]}$ Neuroplasticity is the ability of the central nervous system to respond to internal and external stimuli by reorganizing its structure, function, and connections. ${ }^{[36]}$ Normal ageing is associated with a decline in cognitive function and reduced plasticity. ${ }^{[36]}$ These negative changes can be experienced as reductions in the processing speed, working memory, and peripheral nervous system functions; all of which may be associated with poorer rehabilitation outcomes. ${ }^{[36]}$ Consequently, one should expect a difference between the groups' performance and ability to rehabilitate.

After receiving rehabilitation from a multidisciplinary team, the HIV-positive group was discharged with a mean functional score of 96 points and the HIV-negative group on 97 points. Inconsistencies in the day-to-day treatment were minimized in this study, as all stroke survivors had the same time period and level of treatment intensity on a daily basis. It is also important to note that some functional recovery occurs spontaneously as a result of the resolution of edema in the brain, as well as possible recovery of tissue function in tissues that were ischemic, but not destroyed. ${ }^{[37]}$ Despite the negative effects of HIV and ART drugs on the body and its physical performance, the effect of treatment in helping to achieve immune restoration is effective. ${ }^{[38]}$ Therefore, the fact that a younger population of individuals suffering from HIV, but are 
being treated with ART drugs are relatively stronger individuals to approach the rehabilitation process with more energy and better muscle strength.

During their stay, the patients with HIV improved with an average of 40 points and the individuals without HIV by 38 points. This change in scores appears similar for both groups; however, the individuals without HIV took on average eight-day longer to achieve this increase in points. Again, age can be considered as the decisive factor when analyzing this difference in the length of stay. Neural plasticity is critical for functional recovery and this occurs more effectively in younger individuals. ${ }^{[39]}$ When performing the various t-tests there were, indeed, no significant differences between the two different clinical groups.

Nonetheless, in statistical analysis, the small sample size of HIV-positive individuals in this study did not provide sufficient power against the HIV-negative individuals. The vast difference in numbers of the two groups also has an influence on the generalizability of the results of this study. Briefly, data are for the period 2005 and 2010 which may not be a reflection of the current outcomes.

In conclusion, despite statistically non-significant findings when comparing the HIV-positive and HIV-negative groups' functional outcomes, this study yielded interesting results. There was a considerable difference in the age of stroke onset between the two groups. The HIV-positive group was younger, achieved their increase in score over a period that was 7.5 days shorter than those in the HIV-negative group. We can infer from these findings that stroke survivors who are HIV-positive and are on ART and rehabilitation may recover similar to those who are HIV-negative, spending a similar length of stay in a rehabilitation clinic. Therefore, stroke survivors who are HIV-positive should receive full rehabilitation similar to any other stroke survivors.

\section{Declaration of conflicting interests}

The authors declared no conflicts of interest with respect to the authorship and/or publication of this article.

\section{Funding}

The authors received no financial support for the research and/or authorship of this article.

\section{REFERENCES}

1. Cadilhac DA, Dewey HM, Vos T, Carter R, Thrift AG. The health loss from ischemic stroke and intracerebral hemorrhage: evidence from the North East Melbourne Stroke Incidence Study (NEMESIS). Health Qual Life Outcomes 2010;8:49.
2. Stuart-Shor EM, Wellenius GA, DelloIacono DM, Mittleman MA. Gender differences in presenting and prodromal stroke symptoms. Stroke 2009;40:1121-6.

3. Ogeng'o JA, Olabu BO, Mburu AN, Sinkeet SR. Pediatric stroke in an African country. J Pediatr Neurosci 2010;5:22-4.

4. Nagel MA, Mahalingam R, Cohrs RJ, Gilden D. Virus vasculopathy and stroke: an under-recognized cause and treatment target. Infect Disord Drug Targets 2010;10:105-11.

5. Appelros P, Stegmayr B, Terént A. Sex differences in stroke epidemiology: a systematic review. Stroke 2009;40:1082-90.

6. Saxena S, Ng T, Yong D, Fong N, Kohn G. Functional outcomes in inpatient rehabilitative care of stroke patients: predictive factors and the effect of therapy intensity. Qual Prim Care 2006;14:145-53.

7. Kwakkel G, Kollen B, Lindeman E. Understanding the pattern of functional recovery after stroke: facts and theories. Restor Neurol Neurosci 2004;22:281-99.

8. UNAIDS. The Gap Report Available at: http://www. unaids.org/en/resources/campaigns/2014/2014gapreport/ gapreport/ [Accessed: July 17, 2014]

9. UNAIDS Global report. Available at: http://www.unaids. org/sites/default/files/media_asset/UNAIDS_Global_ Report_2013_en_1.pdf [Accessed: April 20, 2017].

10. UNAIDS. South African HIV estimates. Available at: http:// www.unaids.org/en/regionscountries/countries/southafrica [Accessed: April 10, 2017].

11. Rusch M, Nixon S, Schilder A, Braitstein P, Chan K, Hogg RS. Impairments, activity limitations and participation restrictions: prevalence and associations among persons living with HIV/AIDS in British Columbia. Health Qual Life Outcomes 2004;2:46.

12. Foley J, Ettenhofer M, Wright MJ, Siddiqi I, Choi M, Thames AD, et al. Neurocognitive functioning in HIV-1 infection: effects of cerebrovascular risk factors and age. Clin Neuropsychol 2010;24:265-85.

13. Qureshi AI. HIV infection and stroke: if not protein $S$ deficiency then what explains the relationship? J Neurol Neurosurg Psychiatry 2005;76:1331.

14. Mochan A, Modi M, Modi G. Stroke in black South African HIV-positive patients: a prospective analysis. Stroke 2003;34:10-5.

15. Hama S, Yamashita $H$, Shigenobu $M$, Watanabe A, Hiramoto K, Kurisu K, et al. Depression or apathy and functional recovery after stroke. Int J Geriatr Psychiatry 2007;22:1046-51.

16. Dudgeon WD, Phillips KD, Bopp CM, Hand GA. Physiological and psychological effects of exercise interventions in HIV disease. AIDS Patient Care STDS 2004;18:81-98.

17. Dudgeon WD, Phillips KD, Carson JA, Brewer RB, Durstine JL, Hand GA. Counteracting muscle wasting in HIVinfected individuals. HIV Med 2006;7:299-310.

18. Chestnut TJ. Fatigue in stroke rehabilitation patients: a pilot study. Physiother Res Int 2011;16:151-8.

19. Jacobs GB, Loxton AG, Laten A, Robson B, van Rensburg EJ, Engelbrecht S. Emergence and diversity of different HIV-1 subtypes in South Africa, 2000-2001. J Med Virol 2009;81:1852-9. 
20. Available at: www.sadfm.co.za [Accessed: March 06, 2010].

21. Appelros P, Stegmayr B, Terent A. Sex differences in stroke epidemiology: A systematic review. Stroke 2009;40:1082-90.

22. Glynn JR, Caraël M, Auvert B, Kahindo M, Chege J, Musonda R, et al. Why do young women have a much higher prevalence of HIV than young men? A study in Kisumu, Kenya and Ndola, Zambia. AIDS 2001;15:51-60.

23. The South African Antiretroviral Treatment Guidelines. Available at: http://www.sahivsoc.org/Files/2013\%20 ART\%20Treatment\%20Guidelines\%20Final\%2025\%20 March\%202013\%20corrected.pdf [Accessed: April 10, 2017]

24. Lemogoum D, Degaute JP, Bovet P. Stroke prevention, treatment, and rehabilitation in sub-saharan Africa. Am J Prev Med 2005;29:95-101.

25. Tobias M, Cheung J, Carter K, Anderson C, Feigin VL. Stroke surveillance: population-based estimates and projections for New Zealand. Aust N Z J Public Health 2007;31:520-5.

26. Lee D, Heo SH, Hwa J, Chang D. Stroke subtypes and risk factors of ischemic stroke in young Korean adults. Neurology Asia 2011;16:281-9.

27. Turanjanin N, Jovicevic M, Brkic S, Slankamenac P, Divjak I, Bozic K. Prevalence of risk factors in ischemic stroke patients. HealthMed 2011;5:1273-80.

28. Spengos K, Vemmos K. Risk factors, etiology, and outcome of first-ever ischemic stroke in young adults aged 15 to 45 - the Athens young stroke registry. Eur J Neurol 2010;17:1358-64.

29. Cabral NL, Gonçalves AR, Longo AL, Moro CH, Costa G, Amaral $\mathrm{CH}$, et al. Incidence of stroke subtypes, prognosis and prevalence of risk factors in Joinville, Brazil: a 2 year community based study. J Neurol Neurosurg Psychiatry 2009;80:755-61.
30. Benjamin LA, Bryer A, Emsley HC, Khoo S, Solomon T, Connor MD. HIV infection and stroke: current perspectives and future directions. Lancet Neurol 2012;11:878-90.

31. Young F, Critchley JA, Johnstone LK, Unwin NC. A review of co-morbidity between infectious and chronic disease in Sub Saharan Africa: TB and diabetes mellitus, HIV and metabolic syndrome, and the impact of globalization. Global Health 2009;5:9.

32. Mbanya JC, Motala AA, Sobngwi E, Assah FK, Enoru ST. Diabetes in sub-Saharan Africa. Lancet 2010;375:225466.

33. Rossini PM, Calautti C, Pauri F, Baron JC. Post-stroke plastic reorganisation in the adult brain. Lancet Neurol 2003;2:493-502.

34. Hendricks HT, van Limbeek J, Geurts AC, Zwarts MJ. Motor recovery after stroke: a systematic review of the literature. Arch Phys Med Rehabil 2002;83:1629-37.

35. Teasell R, Bayona NA, Bitensky J. Plasticity and reorganization of the brain post stroke. Top Stroke Rehabil 2005;12:11-26.

36. Cramer SC, Sur M, Dobkin BH, O’Brien C, Sanger TD, Trojanowski JQ, et al. Harnessing neuroplasticity for clinical applications. Brain 2011;134:1591-609.

37. Hallett M. Plasticity of the human motor cortex and recovery from stroke. Brain Res Brain Res Rev 2001;36:169-74.

38. Tan IL, Smith BR, von Geldern G, Mateen FJ, McArthur JC. HIV-associated opportunistic infections of the CNS. Lancet Neurol 2012;11:605-17.

39. Voytek B, Davis M, Yago E, Barceló F, Vogel EK, Knight RT. Dynamic neuroplasticity after human prefrontal cortex damage. Neuron 2010;68:401-8. 